\title{
¿Deben los consultorios jurídicos colombianos transformarse 0 fusionarse con las clínicas jurídicas?
}

\author{
Should Colombian legal \\ services offices be \\ transformed or merged \\ with legal clinics?
}

\section{Gustavo A. Higuita Olaya' \\ gustavo.higuita@upb.edu.co}

https://doi.org/10.22209/rhs.v6n2a04

Recibido: agosto 21 de 2018.

Aceptado: noviembre 26 de 2018.

\section{Resumen}

En los últimos años se ha venido planteando en Colombia la necesidad de realizar una reforma de carácter legal sobre los consultorios jurídicos. Dentro de la baraja de opciones, tal vez la más posible es la de transformarse o fusionarse con las clínicas jurídicas. Incluso la Asociación de Facultades de Derecho (ACOFADE) viene promoviendo la transformación de las prácticas, sugiriendo la posibilidad de acabar con los consultorios, de tal manera que la reforma parece inminente. Es por esto que se considera necesario analizar y cuestionar la viabilidad de los mencionados cambios, reconstruyendo de manera crítica la historia conjunta de las dos instituciones para evitar cometer los defectos que estas han venido presentando. La investigación se realiza

1 Profesor titular de la Universidad Pontificia Bolivariana (UPB) de Medellín. Coordinador del área de Derecho Públicodel Consultorio Jurídico Pío XII de la misma universidad. Miembro del grupo de investigaciones jurídicas -GRID- de la Facultad de Derecho UPB. mediante el análisis de literatura especializada y de mi experiencia profesional como coordinador del área de Derecho Público del Consultorio Jurídico de la Universidad Pontificia Bolivariana. La conclusión defiende la existencia de los dos modelos de manera independiente, propugnando por una reivindicación de los consultorios jurídicos, por medio de un ejercicio autocrítico que les permita reconocer sus fallas históricas, otorgándoles un nuevo carácter emancipatorio y liberador de las ataduras impuestas por el Estado, las facultades de Derecho y la ley.

Palabras clave: Derecho; Educación; Reforma educativa; Law and Development.

\section{Abstract}

In recent years, the need to carry out a legal reform on legal services offices has been raised in Colombia. Among the range of possibilities proposed, perhaps the most feasible is to transform legal services offices or merge them with legal clinics. Even the Colombian Association of Law Faculties (ACOFADE) has been promoting the transformation of law internships, proposing the possibility of eliminating legal services offices, which shows that thereformisimminent. It is thus necessary to analyze and challenge the feasibility of the above mentioned changes, by critically reconstructing the history of both models in order to avoid making the mistakes they have made in the past. The research 
study was carried out by analyzing specialized literature and drawing on the author's professional experience as a coordinator of the Public Law division of the legal services office of Pontificia Bolivariana university. The paper concludes by defending the existence of the two models independently, advocating for a vindication of legal services offices, through a self-critical exercise that allows them to recognize their historical failures, granting them a new emancipatory and liberating character of the constraints imposed by the State, faculties of law and the law itself.

Keywords: Law; education; education reform; law and development.

\section{Introducción}

\section{ecientemente, en el país se ha desatado \\ Suna crisis de institucionalidad debido} a los casos de corrupción de jueces y abogados, impactando especialmente a las Altas Cortes, como la Corte Suprema de Justicia, ya que varios magistrados y exmagistrados son acusados de negociar fallos con el exfiscal anticorrupción (Clavel, 2017). Estos hechos han afectado la confianza de la opinión pública en las instituciones, especialmente en la administración de justicia, por lo que estas han prendido su «radar ético» para encontrar las causas del problema, y han concluido que este radica en la formación de los abogados. Es decir, han dicho que no son las prácticas estatales y judiciales las que contienen el veneno de la corrupción, sino que este se gesta en las facultades de Derecho, por lo que se requiere intervenir en sus programas.

Ejemplo de este ánimo de intervención es el proyecto de ley que pretende crear un examen que deban aprobar los graduados de Derecho para obtener su tarjeta profesional (El Espectador, 2018). Al respecto, el doctor Germán Navas Talero, uno de los representantes a la Cámara que lideran dicho proyecto de ley, dijo:

Uno de los problemas más graves del país es, precisamente, la debilidad de su sistema judicial, derivado en buena medida por la pésima preparación por parte de quienes están egresando de las facultades de Derecho del país. No hay que olvidar que jueces y fiscales también son abogados, y las faltas que estas personas cometen, muchas veces, son ocasionadas por vacíos en su formación (El Espectador, 2018, párr. 3).

En el caso de los consultorios jurídicos, la Asociación Colombiana de Facultades de Derecho (ACOFADE) viene promoviendo una reforma legal que amplíe de manera sustancial los fines, funciones, actividades y competencias de los consultorios (Molina Betancur, 2013, párr. 4). Ello debido a que sus investigaciones arrojan que los estudiantes tienen falta de voluntad generalizada hacia la práctica en el consultorio, prefiriendo experiencias en las que se les ofrezca remuneración; también porque observan que los problemas más importantes tratados en los consultorios no son reflexionados en las aulas, lo que muestra la distancia de estos con las facultades y la academia en general (Molina Betacur, 2016); y debido a que miran con buenos ojos los procesos de otra institución que ha cobrado fuerza en el país: la clínica jurídica. Ante la crisis, y con base en la fuerza de esta otra institución se han generado opciones de modificación de las prácticas académicas. De ahí que la pregunta «¿deben los consultorios jurídicos transformarse en o fusionarse con las clínicas jurídicas para mejorar la educación y la práctica del Derecho en Colombia?» cobre importancia en el mundo académico y jurídico actual. 
Este texto pretende responder a la pregunta a partir del análisis de literatura especializada y de mi experiencia profesional y académica en consultorios jurídicos y clínicas. La tesis que se defenderá será la de la inconveniencia total de cualquiera de las dos opciones formuladas (transformación o fusión).

\section{Materiales y métodos}

Para responder a la pregunta emplearé, en primer lugar, mi experiencia obtenida en los procesos de clínica jurídica adelantados en el Consultorio Jurídico Pío XII de la Universidad Pontificia Bolivariana, complementados con los procesos de formación ofrecidos por la Universidad de Minnesota en convenio con la Agencia Estadounidense para el Desarrollo Internacional (USAID). Además, me valdré de literatura especializada, haciendo énfasis en la investigación liderada por el profesor de la Universidad de los Andes, Daniel Bonilla Maldonado, titulada Justicia de pobres: una genealogía de los consultorios jurídicos en Colombia. Cabe anotar que el texto del profesor Bonilla se erige como el más completo sobre el tema, debido a la escasa bibliografía que trata la materia (Velásquez, 2012, p. 55; Bonilla Maldonado, Recalde \& Luna Blanco, 2017, p. 4).

Para desarrollar el tema abordaré el origen de los consultorios jurídicos y las clínicas jurídicas en Colombia, y la forma en que ambas instituciones han evolucionado con los años; luego, explicaré desde mi experiencia personal el funcionamiento de ambas instituciones en la práctica; habiendo realizado esto, y con un panorama más amplio, realizaré un comparativo de las dos instituciones a partir de su producción académica, sus apuestas educativas, sus métodos de trabajo y los resultados esperados y obtenidos. Con ello se podrán abordar los problemas atinentes a la transformación -es decir, la eliminación definitiva de los consultorios jurídicos y la adopción o imposición de los modelos de clínica jurídica-, para posteriormente analizar la opción referente a la fusión, convivencia o sinergia entre los dos modelos.

Finalmente, realizaré una conclusión de tipo propositivo donde defienda la existencia de ambas instituciones de manera independiente, propugnando por una reivindicación de los consultorios jurídicos por medio de un ejercicio autocrítico que les permita reconocer sus fallas históricas, de modo que estos desarrollen un carácter emancipatorio y liberador de los impedimentos impuestos por el Estado, las facultades de Derecho y la ley.

\section{Orígenes de los consultorios jurídicos $\mathrm{y}$ de las clínicas jurídicas}

Por curioso que parezca, la inspiración, la motivación y el contexto en que surgen en Colombia los consultorios jurídicos y las clínicas jurídicas, específicamente en su modalidad de interés público, son muy similares, ya que ambas instituciones poseen una fuente de inspiración común: el movimiento Law and Development, surgido en la década de los sesenta en los Estados Unidos. Dicho movimiento considera como uno de los elementos claves para el desarrollo de las naciones el fortalecimiento de todas las instituciones que tienen que ver con el Derecho, a saber: la administración de justicia, los jueces, los abogados, las facultades de Derecho y demás entes que faciliten el acceso a la justicia. Al respecto, Bonilla Maldonado, Recalde \& Luna Blanco afirman que: 
El caso de los consultorios jurídicos trasplantados desde Estados Unidos a Colombia hace explícita y fundamenta la teoría funcionalista. Las élites políticas y jurídicas estadounidenses y colombianas decidieron exportar e importar un conjunto de prácticas jurídicas que permitirían enfrentar, en conjunto con otros productos jurídicos, los problemas de justicia social y desarrollo económico que tienen todas las democracias liberales, entre ellas la colombiana. En particular, los consultorios jurídicos contribuirían a enfrentar el déficit en la materialización del derecho a la justicia de las personas de bajos recursos socioeconómicos que tiene Colombia y que Estados Unidos había enfrentado con anterioridad. Del mismo modo, estas instituciones académicas podrían atacar las debilidades de un sistema educativo que reproduce un concepto formalista del derecho, que se había convertido en un obstáculo tanto para la prosperidad económica como para la inclusión plena de todos los miembros de la comunidad política (Bonilla Maldonaldo, Recalde \& Luna Blanco, 2017, p. 338).

Paralelamente al movimiento Law and Development, en los Estados Unidos surgieron y se desarrollaron dos instituciones importantes relacionadas con el proceso colombiano. La primera la podríamos denominar como un servicio de asistencia social o Legal aid, que sirve para ayudar a las personas de escasos recursos a obtener servicios legales, en el que algunos abogados prestan una especie de voluntariado en una organización no gubernamental (ONG), o donan parte de su tiempo para llevar casos de manera gratuita, lo que es conocido como Pro bono. La segunda institución se gestó y desarrolló en las facultades de Derecho y es conocida como Legal clinic, la cual propugna por un aprendizaje práctico del Derecho, basado en la metodología del Learning by doing, es decir, aprender haciendo (Bonilla Maldonado, Recalde \& Luna Blanco, 2013, pp. 31-32).

Con estos antecedentes, hacia 1971 los consultorios jurídicos fueron creados mediante ley en Colombia. Se estableció que las escuelas de Derecho de todo el país deberían organizar, con los estudiantes de los dos últimos años académicos, un tipo de servicio de asistencia legal que requeriría aprobación del respectivo Tribunal Superior del Distrito Judicial para su funcionamiento. Así, los consultorios jurídicos trabajan hoy bajo la dirección de docentes designados o abogados litigantes y deben actuar en coordinación con los estudiantes en los lugares donde el servicio esté establecido. Los estudiantes actúan como abogados para las personas de bajos recursos, condición esta que debe ser probada (artículo 30 del Decreto 196 de 1971). La ley determinó la forma en que el servicio debe ser otorgado y los temas o materias en los que los estudiantes pueden actuar como litigantes.

Por su parte, en la década del 2000, como resultado de las prácticas académicas de las facultades de Derecho y las interacciones entre Colombia y Norte América, las clínicas jurídicas de interés público emergieron en Colombia (Bonilla Maldonado, Recalde \& Luna Blanco, 2017, p. 57). Estas fueron pensadas como espacios para mejorar la educación legal a partir de la transformación de las prácticas de los estudiantes de Derecho, pero estas no aparecieron como una consecuencia de un cambio legislativo o de regulación legal, pues en efecto las clínicas jurídicas no están expresamente regladas en las mismas leyes que regulan los consultorios jurídicos, es más, no tienen ninguna regulación legal alguna.

En este orden de ideas, la motivación y el contexto de ambas instituciones no son diferentes, 
ya que las dos surgen en medio de un ambiente formalista, ritualista e inoperante de las instituciones jurídicas colombianas, como por ejemplo la falta de credibilidad de los jueces y abogados tanto desde el punto de vista de su formación como de su credibilidad moral. En ese ambiente se consideró necesario intervenir de manera clara en los procesos de formación de abogados y jueces, es decir, generar procesos de cambio en la educación del Derecho a través de la implementación de nuevas metodologías de enseñanza-aprendizaje, que impactaran en la formación de los abogados para que estos a su vez propugnaran por la transformación y mejoramiento del sistema.

Pero respecto a la creación sí existen diferencias entre ambas instituciones: una diferencia es de tipo temporal, pues los consultorios jurídicos tal y como los conocemos hoy fueron creados en la década del setenta, mientras que las clínicas jurídicas en su modalidad de interés público son posteriores al año 2000 (Bonilla Maldonado, 2017, pp. 6-8); además, se diferencian en la forma de su creación, ya que los primeros tienen un origen legal, mientras que las segundas surgen a través de convenios con universidades norteamericanas fomentados por la Agencia Estadounidense para el Desarrollo Internacional (USAID) (Bonilla Maldonado, 2013, p. 32).

\section{Mi experiencia}

\section{en el consultorio jurídico y en la clínica jurídica}

Mi experiencia docente se remonta al año 2005, cuando empecé a ejercer como profesor e investigador en varias universidades del país, tanto en pregrado como en posgrado. Durante ese tiempo tuve algunos contactos con el litigio y estuve de manera esporádica en la
Rama Judicial. Lo anterior es importante porque solo hasta el 2013 ingresé al consultorio jurídico de la Universidad Pontificia Bolivariana como coordinador y asesor del área de Derecho Público, lo cual quiere decir que mi perfil y formación no son los tradicionales de los consultorios jurídicos, a saber, abogados litigantes. Ello tiene relevancia en la medida en que esa formación académica hace que mis reflexiones en torno a los consultorios jurídicos presenten matices distintos a los de muchos coordinadores de consultorios, que están más centrados en las ritualidades de la práctica jurídica que en los procesos de formación mismos.

Pues bien, con base en mi experiencia en el consultorio jurídico puedo decir que el trabajo allí es tan intenso por el número de casos, estudiantes, brigadas y demás compromisos que difícilmente hay tiempo o espacio para hacer reflexiones profundas sobre lo que se hace. Pese a lo anterior, lo que más resalta de la labor de los consultorios es la función social desarrollada, entendida esta como la atención a personas de escasos recursos -y en el caso específico del área de Derecho Público, la atención de población vulnerable-, propugnando por la defensa y protección de sus derechos atropellados, en la mayoría de los casos por instituciones estatales. Ello convierte al consultorio jurídico en un valioso mecanismo de ayuda al acceso a la administración de justicia.

El asunto es que, cuando se entiende como prioritario lo social, los consultorios jurídicos suelen enfocar sus objetivos en abarcar un mayor número de población, atender la mayor cantidad de casos posibles, es decir, aumentar y mejorar en todos aquellos indicadores de atención que les permita mostrar unas mejores estadísticas de prestación de los servicios. Lo anterior genera una tensión insalvable con la función 
formativa, es decir, la ateniente a los procesos de enseñanza-aprendizaje, y a la metodología subyacente de aprender haciendo, pues la cantidad de casos y su urgencia difícilmente permiten un desarrollo óptimo de competencias como la escritura, la oralidad, el trabajo en equipo y el pensamiento crítico.

Además, es difícil realizar procesos analíticos de enseñanza a través del estudio de casos difíciles, por lo cual en muchas ocasiones los procesos de enseñanza-aprendizaje se limitan a la explicación de procedimientos o ritos, como lo denominan algunos críticos de los consultorios jurídicos. Lo que digo se corrobora por lo que comentan Bonilla Maldonado, Recalde \& Luna Blanco:

Estos espacios académicos han sido históricamente instituciones que han ayudado a consolidar y reproducir el legalismo y ritualismo comunes en nuestras facultades de derecho y en nuestra administración de justicia (Duque Quintero et al., 2012, p. 293). Los consultorios jurídicos muy poco tiempo después de su creación fueron convertidos en espacios de práctica forense que reflejaban y reforzaban el sistema judicial (Castro, 2014). Las dinámicas de los consultorios han girado alrededor de la asesoría mecánica a los clientes haciendo uso de formatos establecidos para enfrentar los problemas recurrentes de los clientes que comúnmente acuden a estas instituciones para satisfacer sus necesidades jurídicas (Bonilla Maldonado, Recalde \& Luna Blanco, 2017, p. 41).

Ahora bien, paralelamente con mi labor en el consultorio, se desarrollaba la Alianza por los
Derechos Humanos ${ }^{2}$ en convenio con las clínicas jurídicas de la Universidad de Minnesota, la Universidad Pontificia Bolivariana, la Universidad de Antioquia, la Universidad de Medellín y la Universidad Católica de Oriente. Durante este transcurso fui invitado a varios cursos de capacitación con profesores internacionales en técnicas de clínica jurídica. En un primer momento dicha formación me resultó llamativa, porque este nuevo modelo propugnaba por un ejercicio de tipo colaborativo con énfasis en la docencia y la investigación, teniendo como finalidad la formulación de estrategias de litigio de alto impacto que permitieran tener unos mejores resultados.

Por ello comencé a tratar de implementar procesos de clínica en el consultorio jurídico de la Universidad Pontificia Bolivariana. En un primer momento creé grupos de trabajo para que manejaran casos difíciles de manera más eficiente a través del trabajo colaborativo. Esto permitió a mi grupo de trabajo definir y enfocarnos en temas más específicos como los de víctimas de conflicto armado interno, salud, seguridad social y derechos colectivos. Ahora bien, pese a que los resultados de este trabajo se podrían

2 La alianza por los derechos humanos es una iniciativa de tres años de duración destinada a mejorar la educación y la formación en materia de derechos humanos en las escuelas de Derecho y la promoción de una cultura de la aceptación y el respeto de los derechos humanos en Colombia. Financiada por la Agencia Estadounidense para el DesarroIlo Internacional misión en Colombia (USAID/Colombia), soporta tres asociaciones en Antioquia, Valle del Cauca y en la costa del Caribe. En Antioquia el proyecto se realiza entre la Universidad de Minnesota (UMN) y un consorcio de cuatro universidades antioqueñas (Universidad de Antioquia -UDEA-, de la Universidad de Medellín -UDEM-, Universidad Pontificia Bolivariana -UPB-, y la Universidad Católica del Oriente-UCO-). El programa ha sido administrado, apoyado y supervisado de cerca por la High Education for Development, oficina de los Estados Unidos encargada de administrar los recursos de la USAID. 
calificar de manera general como positivos, todavía se estaba muy lejos de las metodologías y los resultados de alto impacto buscados por las clínicas jurídicas.

Por todo ello tomé la decisión de proponer una clínica jurídica oficial del consultorio, aprovechando la coyuntura de que la mayoría de los estudiantes que habían pertenecido a otras clínicas de la facultad -lo que implicaba una formación de años, sumado a la participación en concursos- ingresaban como estudiantes del consultorio. El estímulo para los mismos consistió en ofrecerles un trato de carácter preferencial que les permitiera concentrarse solo en las labores de la clínica, aprovechando las habilidades y conocimientos adquiridos. La metodología propuesta fue que los casos difíciles fueran seleccionados directamente del consultorio y manejados con los conocimientos adquiridos y competencias desarrolladas para lograr -ahora sí- los resultados de alto impacto esperados.

Igual que en el anterior ejercicio, los resultados de este han sido ambivalentes, ya que por un lado se pudo desplegar actividad académica, representada en la presentación de ponencias y la elaboración de conceptos para la Corte Constitucional, pero en lo referente a los casos el manejo no distó mucho de las dificultades propias de los casos adelantados bajo la modalidad de consultorio.

\section{Una caricatura}

\section{de los consultorios jurídicos y de las clínicas jurídicas}

Con base en el marco teórico explicado frente al funcionamiento de los consultorios jurídicos y las clínicas, y a partir de mi experiencia en los consultorios y clínicas jurídicas, a continuación, realizaré un paralelo comparativo entre los consultorios jurídicos y las clínicas jurídicas, tomando en cuenta a los imaginarios sociales creados en las facultades de Derecho y a algunos referentes teóricos, como son los textos referenciados del profesor Bonilla Maldonado y de ACOFADE. Este comparativo será de utilidad a la hora de establecer las consecuencias de la transformación de los consultorios jurídicos en clínicas, o de su fusión entre ellas, pues permitirá ver las consecuencias de ambas alternativas con mayor claridad.

Para realizar el comparativo me basaré en las siguientes seis categorías: la primera es de investigación y producción académica; la segunda es de métodos de acción o funcionamiento; la tercera es de finalidades; la cuarta es de resultados; la quinta es de docentes y la sexta es de estudiantes. Para el comparativo voy a recurrir a una metodología utilizada por el profesor Carlos Santiago Nino para explicar las diferencias entre iuspositivismo e iusnaturalismo: la de caricaturizar las teorías. Esta consiste en exagerar sus rasgos para visualizar mejor sus diferencias, con la salvedad de que en el plano de lo real es poco probable encontrar un esquema que cumpla con todas las características (Nino, 1980).

Comencemos con la primera categoría: investigación y producción académica. En este orden de ideas, si le preguntamos a un consultorio por la investigación y producción académica realizada por él, la respuesta más segura sería «iqué es eso?». Esto se explica porque en casi cincuenta años de creada la institución en Colombia, la investigación y la producción académica de los mismos son prácticamente inexistentes.

Por el contrario, si formulamos la misma pregunta a las clínicas jurídicas, la respuesta será probablemente la petición de que revisemos 
sus papers o su último libro. Esto debido en buena medida a que la investigación hace parte natural de su quehacer, es uno de sus objetivos centrales. De ahí que si miramos la institución en Estados Unidos observaremos que su producción académica es bastante amplia y que incluso si miramos la de Colombia, cuya producción es más modesta (Bonilla Maldonado, 2013, p. 21), la misma es mucho mayor a la referida a los consultorios tanto en términos de cantidad como de calidad. En este sentido la profesora de la Universidad del Rosario, Beatriz Londoño Toro (2016, p. 129), afırma que, en forma paralela al surgimiento de las clínicas en Iberoamérica se observa la necesidad de realizar aportes académicos que se reflejen en las publicaciones de la región.

Dicho lo anterior, prosigamos con la segunda categoría, que es la referente a los métodos: respecto del funcionamiento de los consultorios, puede decirse que en ellos no existe una teoría o método explícito y consciente; simplemente hay formas y rituales, pero propiamente no existe un plan o metodología al llevar los casos. De ahí que algunos investigadores afirman de ellos el que son formalistas, ritualistas, repetitivos y acríticos (Bonilla Maldonado, Recalde \& Luna Blanco, 2017, p. 44).

Por el contrario, las clínicas jurídicas hacen énfasis en sus métodos, como por ejemplo el de enseñanza-aprendizaje, que busca problematizar la realidad, para lo cual se seleccionan casos de alto impacto, se plantea la realización de estrategias de litigio jurídicas y no jurídicas, etc. En este sentido la profesora Londoño Toro plantea que la teoría clínica, la reflexión que la acompaña y la acción que la caracteriza -litigio estratégico- tienen un eje claramente identificable: la justicia social y los derechos humanos (Londoño Toro, 2016, p. 120).
Respecto de la tercera categoría, la de las finalidades buscadas por una y otra institución, podemos decir que lo que pretende el consultorio es por un lado enseñar las ritualidades del sistema judicial, o sea, lo que comúnmente se conoce como «la carpintería del Derecho», y a su vez pretende brindar un servicio social de asistencia a las personas de escasos recursos para que los mismos tengan acceso a la justicia. Las clínicas, por su parte, tienen la finalidad de impactar de manera positiva en el sistema esperando lograr cambios estructurales que les permitan transformar el ordenamiento jurídico y las instituciones.

Frente a la categoría sobre los resultados reales obtenidos hasta el momento, respecto de los consultorios, estos son difíciles de medir por la poca y segregada información que se encuentra sobre los mismos. En efecto, el único dato que podemos considerar relevante es una cifra del 2014 obtenida por la Universidad de los Andes que habla de un número aproximado de 7800 usuarios atendidos mensualmente sumando a todos los consultorios (Bonilla Maldonado, Recalde, \& Luna Blanco, 2017, p. 48), lo cual arroja cerca de 93,600 usuarios atendidos por año. Por su lado las clínicas jurídicas muestran dentro de sus publicaciones resultados referidos a casos relevantes dentro del contexto nacional como, por ejemplo, los del Túnel Verde y la Quebrada la Picacha en Medellín (Por los Derechos Humanos, 2018)³ y otros

3 El caso del Túnel Verde en Medellín ocurrió cuando un grupo ambientalista instauró una acción popular para suspender por el momento la construcción de la sección 2B del sistema masivo de transporte Metroplús, debido a la tala de árboles que esta suponía. La acción fue promovida por una clínica jurídica. Por su parte, en el caso de la Quebrada La Picacha, la quebrada necesitaba ser declarada y tratada como una microcuenca, y debida a la inefectividad de la Administración local, la comunidad vecina estaba sufriendo por los desbordamientos y problemas de salud 
relacionados con temas de discapacidad y defensa de derechos humanos.

Hablemos ahora de la quinta categoría, la de los docentes, bajo una exposición caricaturesca como la que estamos haciendo. Los docentes de consultorio son abogados litigantes de amplia experiencia laboral obtenida en sus propias oficinas, conocedores de los juzgados y de las prácticas formalistas y ritualistas de la administración de justicia; su vinculación con los consultorios es de manera parcial, generalmente por hora cátedra, lo que les permite brindar asesoría a los estudiantes, mientras por otro lado atienden sus negocios jurídicos particulares (Bonilla Maldonado, Recalde \& Luna Blanco, 2017, p. 50). Respecto a las clínicas jurídicas el panorama suele ser distinto, pues en la mayoría de los casos los que las dirigen suelen ser docentes vinculados de tiempo completo, con títulos de doctorado o maestría y con una amplia experiencia docente e investigativa.

Finalmente, respecto a los estudiantes, los que ingresan al consultorio lo hacen de manera obligatoria, reglada por la ley y los respectivos planes de estudio de las universidades, no tienen posibilidad de escoger sus casos, sino que los mismos son impuestos de manera individual, lo cual puede generarles falta de motivación y estrés. Ello arroja como consecuencia que la mayoría realiza una práctica en la que se limita a cumplir con lo impuesto, tal vez no encontrándole ningún beneficio para su proceso de formación y vida profesional.

Por el contrario, la clínica jurídica no es obligatoria, sus estudiantes son seleccionados para

que se generaban. Así que la clínica jurídica de la Universidad de Medellín, en representación de la comunidad, interpuso una acción popular buscando que la Administración desarrollara los proyectos de acuerdo con el plan de microcuencas. ingresar, trabajan en grupo, reciben un largo proceso de capacitación aprendiendo desde la práctica, desarrollando competencias como el pensamiento crítico, la escritura y la oralidad y desplegando sus habilidades en la selección de casos y el litigio estratégico, todo esto con un grado alto de motivación, tanto que se considera que los estudiantes son los principales promotores de la educación legal clínica (Londoño Toro, 2016, p. 139).

\section{Críticas al modelo de clínica jurídica}

A lo largo del texto hemos explorado las diferentes críticas que se le pueden hacer a los consultorios jurídicos, las cuales podemos sintetizar de la siguiente manera: desde el momento en que se crearon los consultorios, a través de la ley, se les otorgó un carácter formalista que iba en oposición a la inspiración, la motivación y el contexto en que se generaron los mismos, a saber, ineficiencia del ordenamiento, poca credibilidad de jueces y abogados. Por ello se vio la necesidad imperante de reformar la educación del Derecho para impactar los sujetos que integran las instituciones y de esta manera transformar todo el sistema para lograr superar las condiciones del subdesarrollo.

La consecuente implementación de la figura, como una práctica forense dirigida por abogados litigantes ajenos a los procesos de investigación y producción académica, generó un proceso de olvido extremadamente rápido de sus orígenes y finalidades, transformando a los consultorios jurídicos en espacios totalmente diferentes a las Legal clinics de los Estados Unidos, orientándose en el mejor de los casos hacia el servicio de asistencia social de personas de escasos recursos. Por ello se convirtieron en 
«abogados de pobres», ubicados en espacios alejados conceptualmente de procesos críticos del Derecho, constituyéndose por el contrario en espacios donde se reproducen y aprenden los formalismos y las ritualidades del sistema judicial sin aspirar a generar ningún cambio.

Respecto a las clínicas, la modesta producción en Colombia poco se ha preocupado por hacer ejercicios críticos sobre su implementación, antes bien, las mismas han tenido un ejercicio de difusión de sus múltiples bondades y los valiosos resultados hasta el momento, mostrando en algunas ocasiones una indiferencia despectiva a los procesos adelantados por los consultorios, tanto que -como se ha explicado- entidades como ACOFADE han planteado el fin de los consultorios y la imposición de las clínicas, sin tener en cuenta los contras de estas.

Es por esto que a partir de mi experiencia y de lo comentado por el profesor Bonilla Maldonado, me permitiré enarbolar algunas críticas a las clínicas jurídicas. La primera y tal vez la más evidente, pero ignorada casi de manera sistemática, es referente al trasplante legal, fruto del nuevo proceso de colonización jurídica por parte de los Estados Unidos, que consiste en su creciente influencia en el ordenamiento jurídico, la administración de justicia y la educación del Derecho, promovida, entre otros, por la Agencia de Cooperación Norteamericana (USAID). Ello porque, pese a todas las maravillas propugnadas hacia las clínicas, la adopción o no de las mismas no resulta ser un proceso fortuito, sino que el mismo hace parte de un plan elaborado de imposición de unos estándares norteamericanos, porque el modelo de intervención del movimiento Law and Development, referido al trasplante de las legal clinics a los países de América Latina ha ignorado el entorno, el contexto de las figuras o instituciones ya establecidas y las adaptaciones necesarias en los centros de recepción, haciendo que este en realidad no parezca la búsqueda del desarrollo autónomo de los países, sino la imposición de un estándar estadounidense.

Precisamente, en la medida en que el primer trasplante (el de los consultorios jurídicos) fue realizado de manera acrítica, la consagración legal de la figura hizo que este perdiera sus objetivos iniciales, convirtiendo a los consultorios en una forma de trasladar las obligaciones estatales a entes privados. Pues bien, a pesar de este precedente, las clínicas han sido vistas como una idea novedosa -que no es tal, debido a su origen similar al de los consultoriosque, por ende, debería ser regulada legalmente para implementarse. Este interés por regularlas desconoce los problemas del primer trasplante, ocurridos justamente en razón de su consagración legal, lo cual pone en peligro el funcionamiento de la clínica como mecanismo de formación real para los estudiantes de Derecho.

La segunda critica es que la metodología y el enfoque académico e investigativo de la clínica plantea procesos a largo plazo -por ejemplo, los estudiantes se pueden demorar seis meses o más escogiendo un caso y las estrategias planteadas muchísimo tiempo más-, por lo cual es difícil que un estudiante logre estar en todo el proceso de un caso. Una solución alternativa es plantearse casos hipotéticos, para dominar la técnica y así pasar de una manera más eficiente al manejo de casos reales. Pero esta solución sigue presentando problemas en la medida en que esos casos reales no podrían ser casos comunes como los que llevan los consultorios y mucho menos urgentes como una tutela por salud. De ahí que, si bien es cierto que las habilidades desarrolladas por los estudiantes de clínica son más amplias e incluso 
mejores que las de los estudiantes de consultorio, en el manejo de estos casos «simples» dichas habilidades no marcan ninguna diferencia, es más, a veces el exceso de reflexión en algunos casos se convierte en falta de practicidad y agilidad para responder a las mismas.

\section{Balance final: análisis de las posibilidades de fusión o transformación}

Es momento de volver sobre la pregunta objeto del presente texto: ¿deben los consultorios jurídicos transformarse en o fusionarse con las clínicas jurídicas para mejorar la educación y la práctica del Derecho en Colombia? La respuesta después del análisis presentado es que no, porque la transformación desconocería el punto más valioso de los consultorios, que es su servicio de asistencia social, ya que esto es algo que el modelo de clínica no puede suplir siendo consecuente con su propia metodología. Al respecto nos recuerda el profesor Velásquez lo siguiente:

La calidad de abogados de pobres que ostentan los estudiantes de derecho en sus prácticas de consultorio jurídico por mandato del art. $1^{\circ}$. de la Ley 583 de 2000 , constituye una especie de vocación social que viene impuesta por el legislador desde hace 40 años, inicialmente por el Decreto 196 de 1971, artículo 30, el cual posteriormente fue reformado por la Ley 583 de 2000, norma esta que hizo aún más explícito el mencionado mandato. Dicha calidad contiene un énfasis social que está en perfecta consonancia con el carácter de servicio social y proyección social que en las instituciones universitarias tienen las prácticas, circunstancia que hace que esta directiva legal haya sido aceptada con beneplácito por las instituciones universitarias (Velásquez, 2012, p. 53).
La fusión tampoco se considera adecuada, ya que, bajo la experiencia vivida, los estudiantes formados en las clínicas no logran realmente desplegar sus competencias con los casos del consultorio, y a su vez tampoco desarrollan las competencias y valores propiciados en los mismos. En términos reales dicha fusión sería solo formal; es decir, una regulación legal compartida, y en la práctica estarían juntos, pero no revueltos, de modo que el sometimiento legal bajo la lógica histórica planteada es lo peor que les podría pasar a las clínicas jurídicas, pues sería cometer los mismos errores que se cometieron con los consultorios.

\section{Discusión}

\section{Reivindicación de los consultorios}

Los consultorios deben iniciar un difícil proceso de autorreflexión histórico, que les permita identificar y aceptar los principales errores cometidos durante casi cincuenta años, entre ellos su silencio casi absoluto, reflejado en la falta de investigación, producción académica, consolidación y análisis de cifras, que permitan entender y evaluar realmente sus resultados.

Además, es necesario cerrar las brechas existentes entre los consultorios y las facultades de Derecho para reivindicar el rol fundamental de los consultorios en la compresión de la realidad del sistema judicial y del Derecho en Colombia, generando verdaderos canales de diálogo con las cátedras, que permitan a los estudiantes tener una visión más amplia y de conjunto de los problemas jurídicos, más allá de las teorías segmentadas y compartimentadas dominantes en los planes de estudio académicos. 
Además, es indispensable reivindicar y defender por convicción propia -y no por la mera obligación impuesta de presentar informesla valiosa función social desempeñada por los consultorios, esperando que esta sea respaldada con cifras concretas, segregadas y analizadas, ojalá acompañados con estudio de casos que permitan replantear teorías.

Pero tal vez, sobre lo que más se tienen que cuestionar los consultorios es sobre sus relaciones de sometimiento frente al Estado, específicamente respecto a leyes impuestas por este, pues el Estado les impone unas funciones que le son propias, pero que él mismo es incapaz de realizar, y pese a esto ese mismo Estado se atreve a regularlos, controlarlos y sancionarlos. Por ello es necesario otorgar a los consultorios un nuevo carácter emancipatorio y liberador de los yugos impuestos por la ley, esa ley que para muchos ha sido una zona de confort y una excusa para mantener el orden establecido.

\section{Libertad de las clínicas}

Por su parte, las clínicas deben seguir adelante con sus procesos y no perder el terreno ya ganado en las facultades de Derecho, preocupándose de no cometer los mismos errores que los consultorios, en especial, aceptar de manera pasiva las imposiciones legales y regulatorias, mismas que lejos de convertiste en una victoria que asegure la obligación de la enseñanza clínica, les imponga cargas y métodos que terminen por desnaturalizarlas. En otras palabras, no sería adecuado imponer una regulación legal a las clínicas, dada la experiencia histórica con los consultorios, por lo cual es vital mantener su independencia, luchando con todos sus conocimientos y capacidades para conservar su libertad.

\section{Agradecimientos}

Agradezco a las doctoras Cathalina Sánchez Escobar, Adiley Carmona Montoya y Elvigia Cardona Zuleta, y a la estudiante Laura Arteaga Arango por sus lecturas y comentarios sobre este escrito. Agradezco, también, a todas las demás personas que con sus revisiones y comentarios me guiaron en la realización de este texto. ${ }^{4}$

\section{Referencias}

Bonilla Maldonado, D., Recalde, G., \& Luna Blanco, T. (2017). Justicia de pobres: Una genealogía de los consultorios jurídicos en Colombia. Revista de Derecho (47), 1-72. Obtenido de http://rcientificas.uninorte.edu.co/index.php/derecho/article/ view/8686/9772

Bonilla, D. (2013). Legal Clinics in the Global North and South: Between Equality and Subordination - An Essay. Yale Human Rights and Development Law Journal, 16(1), 1-41.

Clavel, T. (21 de Septiembre de 2017). Colombia Judicial Corruption Scandal Expands With Fmr Top Judge's Arrest. Obtenido de InSight Crime: https:// www.insightcrime.org/news/brief/colombia-judicial-corruption-scandal-expands-fmr-top-judge-arrest/

4 Ideas contenidas en este artículo fueron presentadas en los siguientes eventos durante el 2018. IV Encuentro Regional de la Red de Consultorios Jurídicos y sus Centros de Conciliación de Antioquia: Expectativas vs Realidades en la práctica de los consultorios jurídicos y sus centros de conciliación; VII Encuentro Nacional de Consultorios Jurídicos y sus Centros de Conciliación: La ética en el profesional, una necesidad actual; XI Encuentro del Nodo Antioquia de la Red de Grupos y Centros de Investigación Jurídica y Sociojurídica (Red Sociojurídica); XVIII Encuentro Nacional de la Red Sociojurídica y Annual Meeting on Law and Society de Toronto. 
Colombia, Presidencia de la República. Decreto 196 del doce (12) de febrero de 1971, «Por el cual se dicta el estatuto de la abogacía».

El Espectador. (9 de abril de 2018). Estudiantes de Derecho deberán aprobar un nuevo examen para poder graduarse como abogados. Obtenido de El Espectador: https://www.elespectador.com/ noticias/educacion/estudiantes-de-derecho-deberan-aprobar-un-nuevo-examen-para-poder-graduarse-como-abogados-articulo-749127

Londoño Toro, B. (2016). Los cambios que requieren las clinicas juridicas iberoamericanas. Estudio de caso en seis paises de la región. Boletin mexicano de derecho comparado, 49(146), 119-148.

Molina Betancur, C. M. (10 de marzo de 2016). ¿El fin de los consultorios jurídicos en las facultades de Derecho? Obtenido de Ambito Jurídico: https:// www.ambitojuridico.com/noticias/academia/ educacion-y-cultura/el-fin-de-los-consultorios-juridicos-en-las-facultades-de
Molina Betancur, C. M. (12 de diciembre de 2013). Las prácticas judiciales en las facultades de Derecho. Obtenido de Ámbito Jurídico: https:// www.ambitojuridico.com/noticias/academia/ educacion-y-cultura/las-practicas-judiciales-en-las-facultades-de-derecho

Nino, C. S. (1980). Introduccion al analisis del derecho. Buenos Aires: Astrea.

Por los derechos humanos (s.f.). Historia del pleito jurídico por la Quebrada La Picacha. Obtenido de Por los derechos humanos: https://www. porlosderechoshumanos.com/historia-de-laquebrada-la-picacha-contada-por-la-clinicajuridica-de-udem/

Velásquez, H. (2012). El trabajo social de los consultorios: ¿necesidad u obstaculo? Revista de la Facultad de Derecho y Ciencias Políticas de la Universidad Pontificia Bolivariana 42(116), 51-76. 